\title{
Acousto-optic frequency switching for single-camera planar Doppler velocimetry
}

\author{
Helen D. Ford*, David S. Nobes and Ralph P. Tatam \\ Optical Sensors Group, Centre for Photonics and Optical Engineering, School of Engineering, \\ Cranfield University, Cranfield, Bedford, MK43 0AL, UK.
}

\begin{abstract}
A single-camera Planar Doppler velocimetry system has been demonstrated, in which illumination beams at two closelyspaced optical frequencies are derived from a single Argon-ion laser. The frequency of one beam lies on an absorption line of iodine vapour, and the other just off the absorption line. The beams sequentially illuminate a plane within a seeded flow and the Doppler-shifted scattered light passes through an iodine cell onto a single solid-state camera. Light scattered from the measurement plane from the beam with its wavelength set to be off the absorption line is not affected by its passage through the cell, and provides a reference image, while that from the beam on the absorption line encodes the velocity information as a variation in transmission dependent upon the Doppler shift. The complex imaging system required for conventional PDV is eliminated, since superposition of the reference and signal images is automatic.

The two beams are generated by an optical arrangement incorporating an acousto-optic modulator, and are transmitted to the region of interest by a single mode optical fibre, which ensures both a smooth Gaussian illumination profile and colinearity of the beams. The system is demonstrated using a spinning disc and a velocity resolution of $+/-1 \mathrm{~ms}^{-1}$ is achieved.
\end{abstract}

Keywords: planar Doppler velocimetry, flow measurement, acousto-optic frequency switching

\section{INTRODUCTION}

Planar Doppler velocimetry (PDV) is a flow measurement technique that provides velocity information over a plane defined by a light sheet formed from an expanded laser beam ${ }^{1,2,3}$. The optical frequency of light scattered from each particle in the seeded flow experiences a Doppler shift, which is linearly related to the velocity of the particle at that point in the flow. In PDV, a portion of the light sheet is imaged, through a glass cell containing iodine vapour, onto the active area of a solid state camera. Iodine has a number of narrow absorption lines over the visible region of the spectrum ${ }^{4,5}$. If the laser frequency is chosen to coincide with one such line, the optical intensity at any position in the camera image is a function of the Doppler shift experienced at the corresponding flow position, via the frequency-dependent iodine absorption.

The intensity over the image varies for reasons other than the iodine absorption. The laser sheet typically has a Gaussian intensity profile, seeding density is not uniform and diffraction fringes caused by imperfections in the optical surfaces can appear in the images. To eliminate these variations, it is usual to amplitude-divide the image beam onto either two cameras ${ }^{6}$ or two halves of a single camera ${ }^{7}$, as shown in figure 1 (a) and (b). From one of the two imaging paths the iodine cell is omitted, and the resulting image acts as a reference to normalise the signal image carrying the velocity information.

If accurate velocity information is to be obtained, the signal and reference images must be identical, as far as possible, apart from the effect of the iodine absorption. Even very slight differences in the magnification or the optical distortions of the two images can lead to significant errors in the calculated velocity. Superposition of the two images must be achieved to sub-pixel accuracy, particularly if large velocity gradients are present in the region imaged. Software de-warping routines can achieve good pixel matching and can help to correct for small differences in magnification ${ }^{8,9}$. Post-processing cannot, however, correct for the almost inevitable differences in the optical distortions, although these could be minimised by including a dummy cell in the reference arm of the system. The beamsplitting cube that is typically used to divide the image can also introduce errors. The splitting ratio of this component, even if it is described as a non-polarising cube, will generally have some polarisation sensitivity that can affect the intensities seen by the two cameras.

* h.d.ford@ cranfield.ac.uk; phone +44 (0)1234 750111 x5345; fax +44 (0)1234 752452; http://www.cranfield.ac.uk/sme/cpoe/osg.htm 


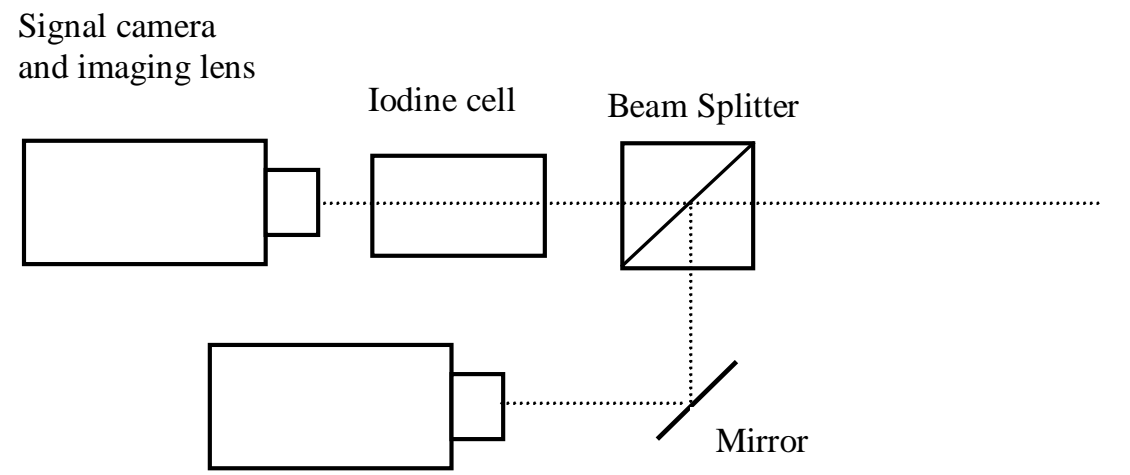

(a)

Reference camera

and imaging lens

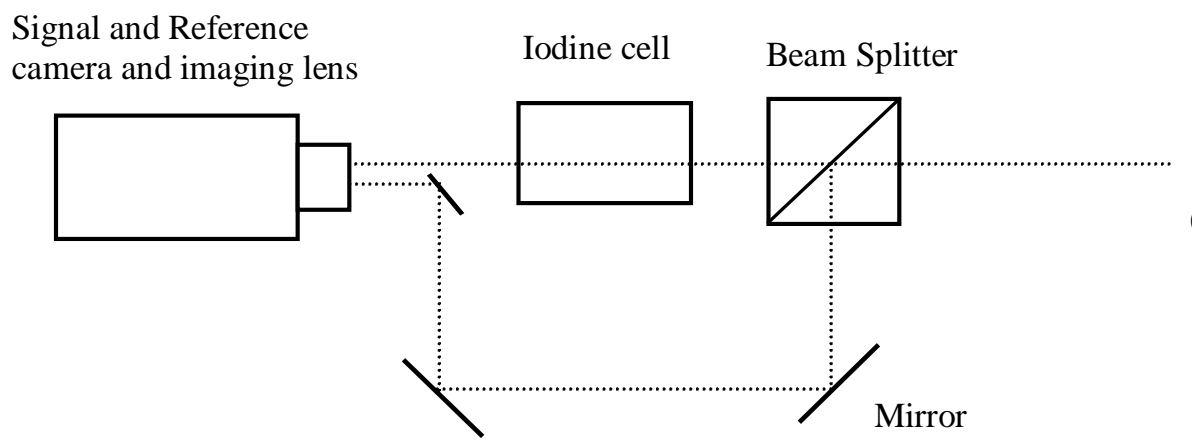

(b)

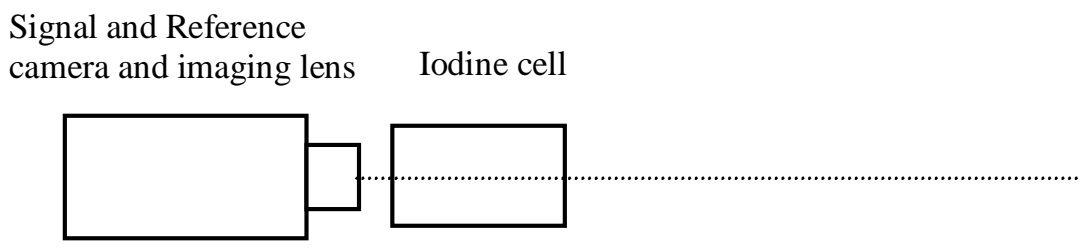

(c)

Figure 1: Schematics of typical PDV image-capture arrangements; (a) two-camera system, (b) single-camera split image arrangement, (c) single-camera dual-frequency arrangement.

The technique described in this paper offers one solution to the pixel-matching problem. A portion of the laser beam used to illuminate the flow is made to pass through an acousto-optic modulator (AOM). This has the effect of slightly altering the optical frequency of the light. The laser is tuned just off the low frequency side of the absorption line and the AOM is chosen to produce a frequency upshift of $260 \mathrm{MHz}$, so that a double pass through the device results in a total upshift of 520 $\mathrm{MHz}$. This is sufficient to take the optical frequency from the original position off the side of the iodine absorption line to a position close to the $50 \%$ absorption level on this side of the line. Since the original laser frequency is off the absorption line, this beam will pass unaffected through the iodine cell. Thus, illumination beams appropriate for acquisition of both reference and signal images can be derived from the same source, and a common path optical geometry becomes possible, 
where the PDV system is reduced simply to an iodine cell positioned directly in front of the lens of the solid-state camera, as shown in figure 1 (c).

The reference and signal illumination beams diverge from a common point source, defined by the core of the optical fibre used for beam delivery, and have identical beam profiles. Furthermore, since the wavelength difference between the two beams is negligible in comparison with the typical size of scattering particles, the scattering efficiency should be identical for the two beams at any particular orientation. Exact alignment of the reference and signal images on the active area of the camera is automatic.

The disadvantage of the system described above is that simultaneous image acquisition is no longer possible. Since the reference and signal images are not spatially separated, they must instead be separated in time. For steady-state flows this is not a major difficulty, provided that the seeding is relatively dense and the seeding distribution remains essentially unchanged during the time taken to acquire the two image frames. The extent to which this condition is satisfied will depend upon the individual flow situation. The accuracy of measurements made in a steady-state flow can, of course, be improved by acquiring a succession of image pairs and taking an average of the normalised intensity maps over an extended period.

A two-colour PDV system, using a single camera has previously been described ${ }^{10}$. This system used a reference beam in the red at $618 \mathrm{~nm}$ and a signal beam in the green at $532 \mathrm{~nm}$. A colour camera was used to image the light sheet in the flow, so that the reference and signal images could be obtained simultaneously by the different colour camera channels. However, a large amount of processing was necessary to correct for 'bleed' between colour channels, and data interpolation was required to obtain spatially overlapping images. It is not clear whether the beam profiles for the two colours were matched, or whether account was taken of the significant differences in scattering as a function of geometry, that would be expected for such widely differing wavelengths.

\section{METHOD}

\subsection{Illumination system}

The experimental arrangement of figure 2 was used to generate co-linear beams at two closely spaced optical frequencies for illumination of the single-camera PDV system. The optical components were mounted on a rigid $1 \mathrm{~m}$ by $0.5 \mathrm{~m}$ aluminium plate for portability. In this arrangement, the linearly polarised beam from the laser source is amplitude-divided by a polarising beam splitter (PBS1). The orientation of a half wave plate (HWP) positioned before this beam splitter can be altered to change the splitting ratio, which was set to be about 70/30, the smaller fraction of the light being transmitted in the straight through direction. The straight-through beam is simply redirected using a mirror and a further beamsplitter (PBS2), before being coupled into a single-mode optical fibre. The half-wave plate, orientated so as to rotate the polarisation azimuth through $90^{\circ}$, is necessary to achieve reflection of the beam at the second beam splitter. The light that emerges from the optical fibre is a spatially filtered beam with an approximately Gaussian intensity profile.

The polarisation state of the beam reflected at PBS1 is orthogonal to that of the straight-through beam. This beam is redirected by a third polarising beam splitter (PBS3) and passes through a telescope arrangement, reducing the diameter of the beam by a factor of about three. The reduced beam diameter is sufficiently small to allow the beam to pass through the active area of an AOM positioned in the system, by which the optical frequency is increased by $260 \mathrm{MHz}$. The efficiency of the AOM at this wavelength is about $70 \%$, thus about a third of the power is lost in the frequency conversion. The shifted beam is then retroreflected by a mirror, making a double pass through a quarter wave plate (QWP), which rotates the polarisation state by $90^{\circ}$. The beam makes a second transit through the AOM, doubling the optical frequency shift to a total of $520 \mathrm{MHz}$ with a further reduction in power, and is recombined with the reference beam in PBS2 and coupled into the optical fibre. At the point of recombination, the optical power in each of the two beams is similar. Careful alignment is required to maximise the power coupling from both beams simultaneously into the fibre. The optical fibre is birefringent ${ }^{11}$, and is orientated such that the polarisation states of the two input beams are aligned with the polarisation axes of the fibre. This ensures that the linear polarisation states of the two beams are preserved as they propagate down the fibre. To minimise scattering variations caused by the different polarisations of the two illumination beams, an appropriately orientated quarter wave plate at the fibre output converts the orthogonally polarised linear states into orthogonal circular states. Cylindrical optics can then be used to form the circular Gaussian beam into a light sheet. 


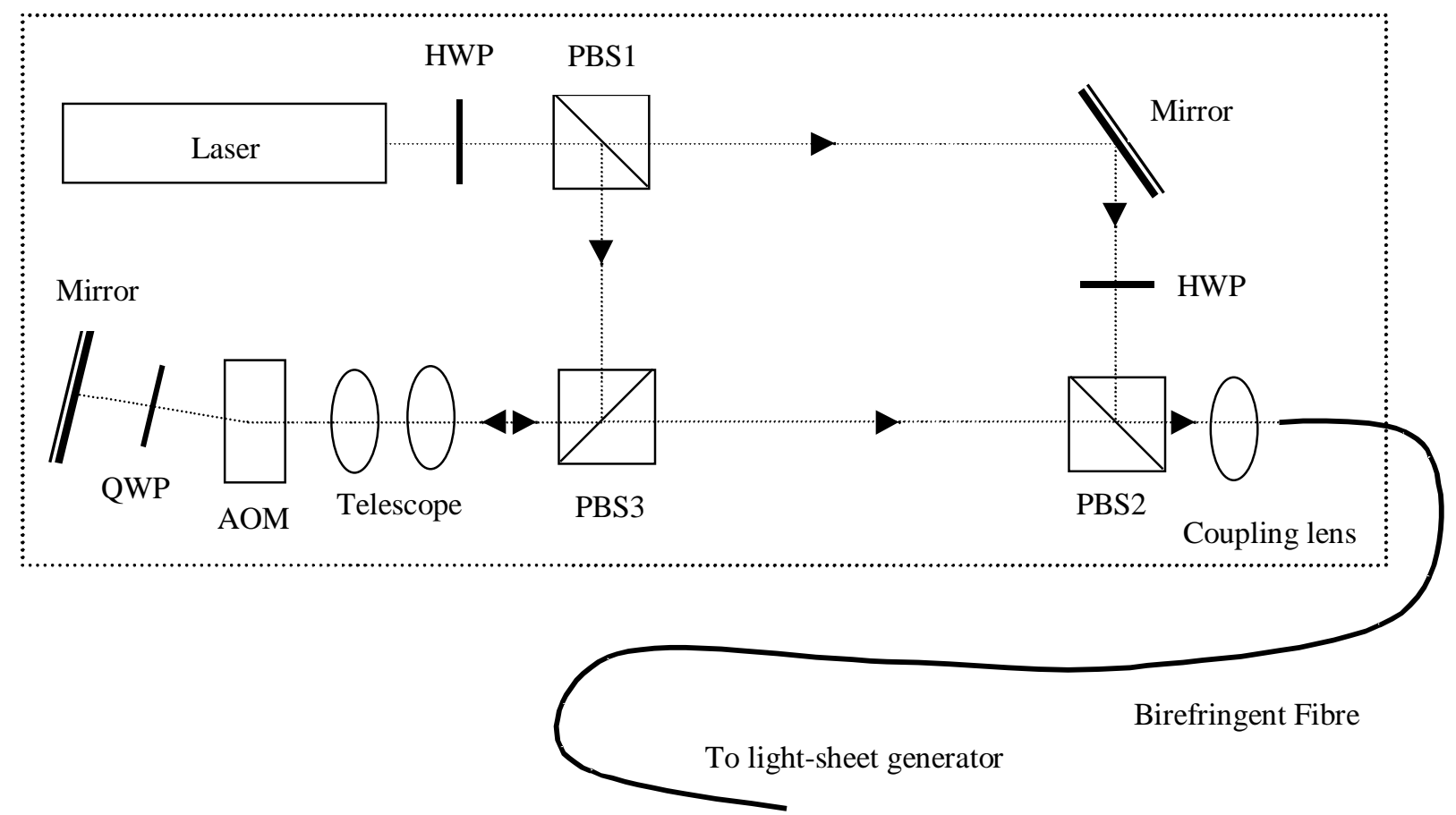

Figure 2: Experimental arrangement for the generation of two optical frequencies spaced $520 \mathrm{MHz}$ apart.

The light source was an Excel Argon-ion laser, incorporating a temperature-stabilised etalon to ensure single-mode operation at $514.5 \mathrm{~nm}$. The single-mode linewidth was about $3 \mathrm{MHz}$. Although this laser was not designed as a tunable source, the etalon temperature could be altered in rather a coarse manner, using a potentiometer on the rear of the laser. The setting resolution of this control was about 70-100 MHz. The iodine cell itself was used to monitor the frequency variation and to locate the absorption line, since no indication of the optical frequency was available from the laser electronics.

During the experiments described in this paper, the laser wavelength is tuned just off the iodine absorption line, and the unshifted beam is subsequently used to obtain reference images in the PDV system. Illumination from this beam, which bypasses the AOM, is permanently "on", and is present in all images captured by the PDV camera system. The frequency of the upshifted beam automatically lies at a position somewhere on the low-frequency side of the iodine absorption line. This beam, which is present at the fibre output only when the AOM is switched on, is used to obtain the signal images in the PDV system. Both signal and reference beams experience scattering from the flow, and the frequency shifts imposed on each beam are identical. Since both beams also pass through the iodine cell, the initial reference frequency must be positioned sufficiently far from the top of the absorption line that the maximum Doppler upshift expected in the system will not bring it down below the top of the line.

\subsection{Image-capture system}

As described in the introduction, the image-capture system is greatly simplified compared with that required for a single frequency of illumination. Both reference and signal beams pass through the iodine cell, and the system is reduced to a single solid state camera incorporating a zoom lens, together with a temperature-stabilised iodine cell positioned directly in front of the lens.

The camera used is an Imager 3 supplied by LaVision. It is a digital camera with 12 bit A/D conversion on a Peltier-cooled chip, and $1280 \times 1024$ image resolution. Dedicated image acquisition and processing software (DaVis 6.0) is used to control the camera and to calculate and display normalised intensity maps. The iodine cell, which operates as a saturated cell ${ }^{12}$, is $25 \mathrm{~mm}$ in diameter and $50 \mathrm{~mm}$ long, with a cold finger. The cold finger is held at $40^{\circ} \mathrm{C}$ using a Peltier element in a feedback loop, and the cell body is contained in an oven held at $56.5^{\circ} \mathrm{C}$. 
Accurate velocity measurements using PDV require an exact knowledge of the shape of the iodine absorption line as a function of frequency. The tuning method for the laser described above did not have sufficient resolution to allow the form of the absorption line to be measured. This measurement was made independently using a Spectra-Physics Argon-ion laser with a highly stabilised etalon and frequency-scanning capability. The resulting plot of iodine transmission as a function of optical frequency is shown in figure 3 , together with a sixth-order polynomial fit.

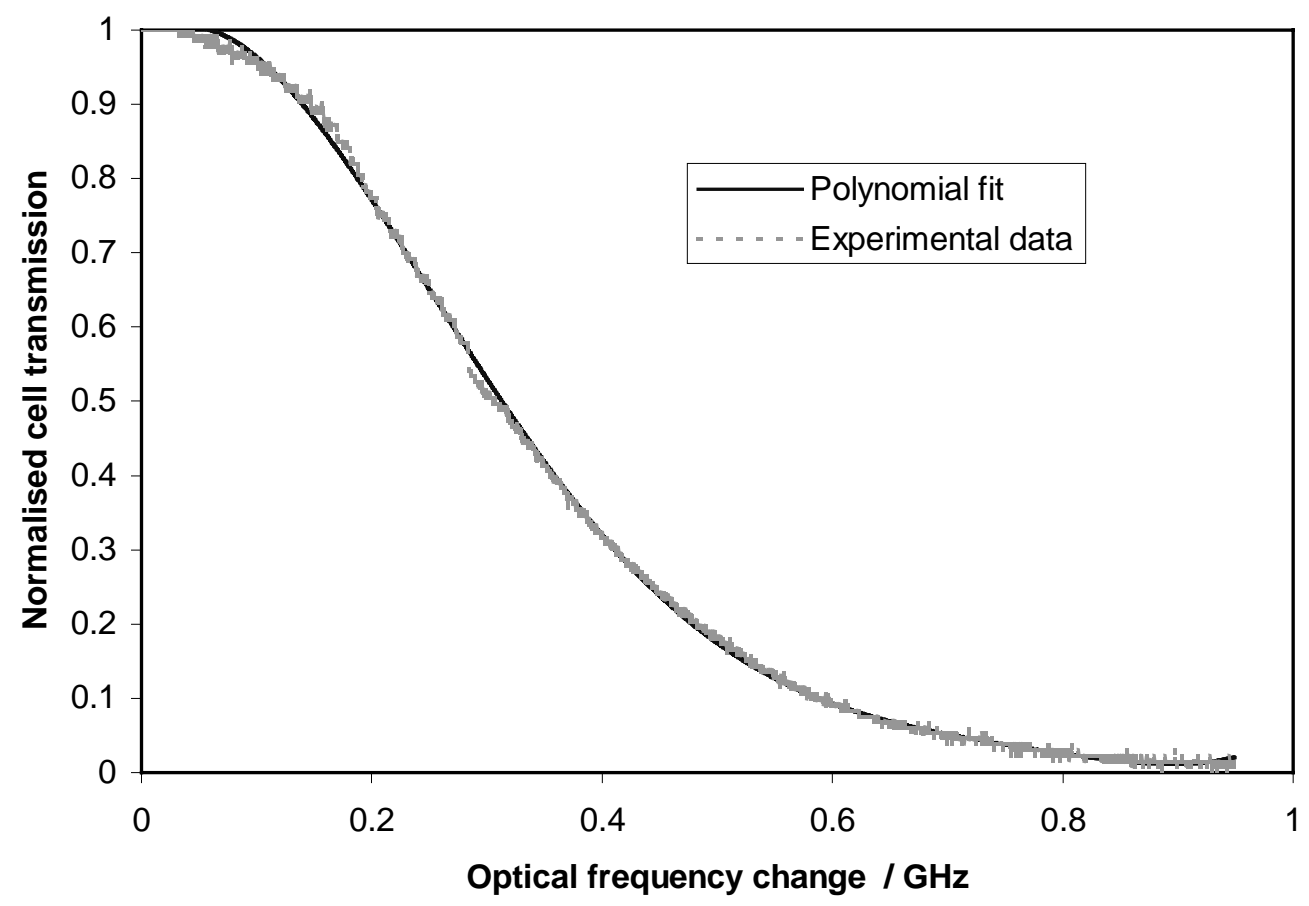

Figure 3: Plot showing the normalised transmission of the iodine vapour cell as a function of laser frequency (dotted line), and a sixth-order polynomial fit to the data (solid line).

The AOM in the illumination system can be switched on or off by a TTL signal sent to the driver. When the TTL signal goes high, the AOM is on, and upshifted light is present at the fibre output. Synchronisation of image capture with the appropriate illumination beams was achieved by programming the camera to send out a TTL signal to the AOM prior to each operation of the electronic shutter. Pairs of images were collected and stored, initially in memory, since this allowed a large number of image pairs to be acquired in rapid succession. In these experiments, 40 image pairs were acquired and stored in about 15 seconds, using a camera exposure time of $100 \mathrm{~ms}$. The relatively slow step of saving the data onto the hard disc was performed only after acquisition of all images in the set had been completed.

Typically, in PDV, one of each pair results purely from illumination by the reference beam and the other purely from illumination by the signal beam. The ratio then produces a normalised intensity map. However, in our system, the reference beam was always present. The resulting pairs of images thus comprised one frame illuminated purely by reference (unshifted) light and the other by reference plus signal (shifted) light. The processing required to obtain a normalised intensity map now involves an extra step; one image must first be subtracted from the other to extract the effect of the signal illumination alone, before taking the usual ratio.

The test object used to assess the PDV system was a perspex disc $150 \mathrm{~mm}$ in diameter, coated with mat white paint and mounted centrally on the spindle of a rotary motor such that the plane of the disc was orthogonal to the plane of the optical bench. The maximum circumferential velocity of the wheel was $31 \mathrm{~ms}^{-1}$. Figure 4 shows a plan view of the experimental arrangement. The geometry is chosen to have high sensitivity to the horizontal component of rotational velocity in the plane 
of the wheel. For measurements on the disc, it was not necessary to form the diverging illumination beam from the fibre into a light sheet, since the front surface of the disc itself defined the measurement plane.

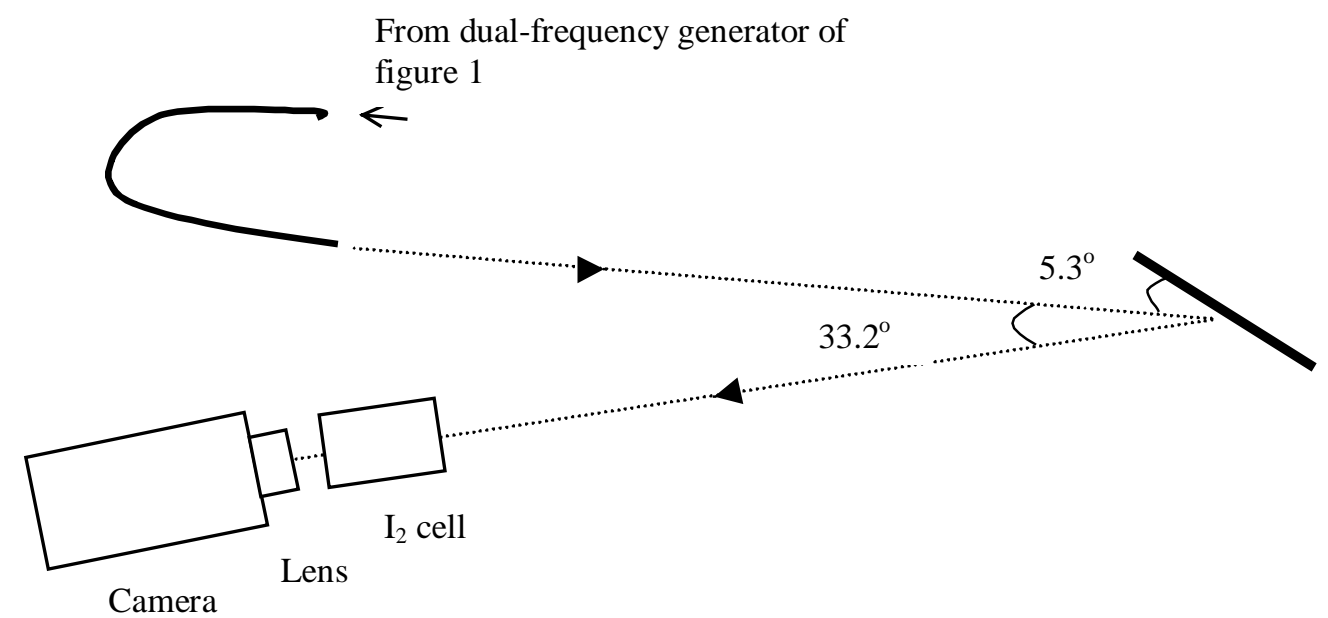

Figure 4: Experimental illumination and observation arrangement.

The expected frequency shift is given by the usual Doppler equation ${ }^{13}$

$$
\Delta v=\frac{v(\overline{\mathbf{o}}-\overline{\mathbf{i}}) \cdot \mathbf{v}}{c},
$$

where $V$ is the optical frequency, $\overline{\mathbf{o}}$ and $\overline{\mathbf{i}}$ are unit vectors in the observation and illumination directions respectively, $\mathbf{v}$ is the velocity vector and $\mathrm{c}$ is the speed of light in air. For the geometry shown in figure 4, a maximum frequency shift of $+/-$ $104 \mathrm{MHz}$ is predicted at the highest and lowest points on the spinning disc, where the velocity vector is horizontal. The horizontal component of velocity should vary linearly along any vertical line through the disc.

Background images were acquired by positioning a small circle of card close to the output end of the optical fibre, to throw the surface of the wheel alone into shadow while retaining any scattering from surrounding objects that would also be present during velocity measurements. Baffles of black card were arranged to reduce unwanted scattering as much as possible. Background subtractions were performed routinely on all images subsequently acquired by the camera.

\section{RESULTS}

Sets of image pairs were stored for both anticlockwise (series A) and clockwise (series B) rotation of the disc, as viewed from the front, at the maximum rotation speed. For each direction of rotation, 40 image pairs were acquired, the AOM being synchronised with the camera shutter to provide the appropriate illumination for each image. The images were saved to disc as pairs: image $\mathrm{RS}=$ reference + signal illumination and image $\mathrm{R}=$ reference illumination for each pair. A typical image pair is shown in figure 5 , for anticlockwise rotation of the disc. 

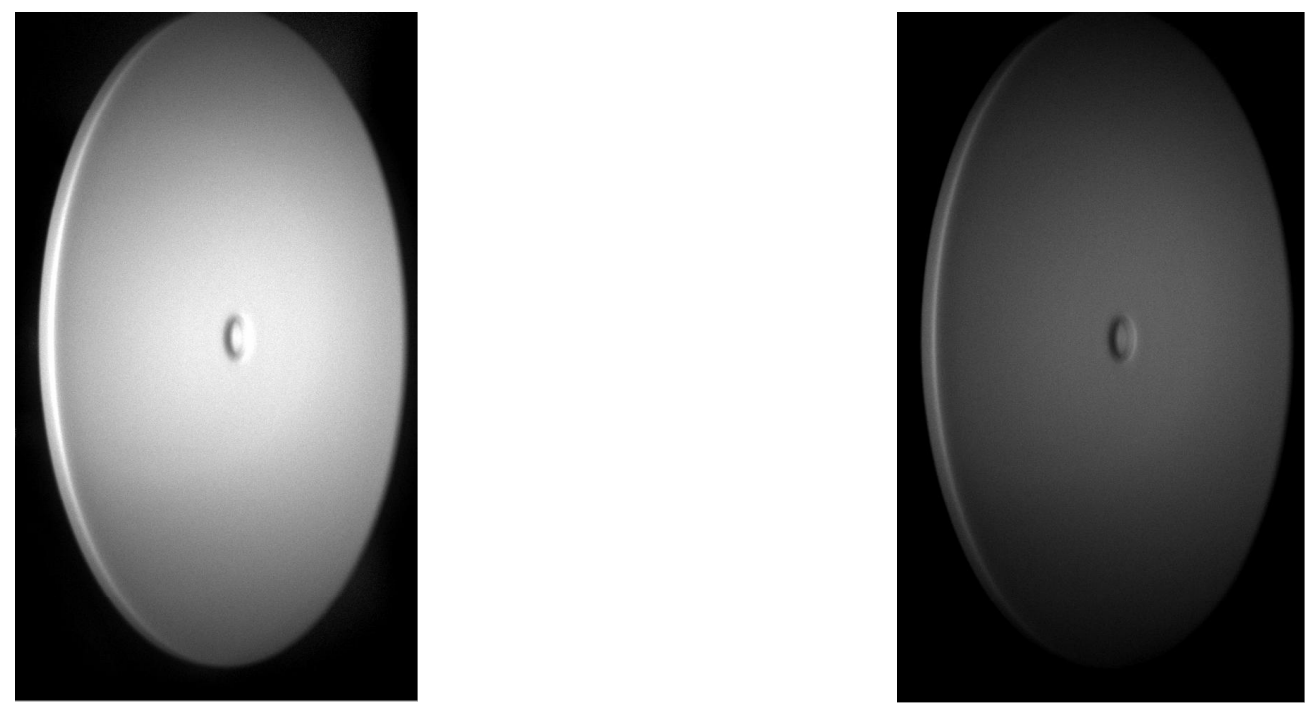

Figure 5: A typical image pair (number 20 of the 40 images stored) acquired by the camera for anticlockwise rotation of the disc; (a) image $\mathrm{R}+\mathrm{S}$, (b) image $\mathrm{R}$.

In the images, the intensity profile of the illumination beam results in high greyscale values for pixels near the centre of the disc, and lower values towards the edges of the disc. The initial image analysis, which is carried out using the DaVis 6.0 software by loading the stored images into memory buffers, is simply a subtraction $\mathrm{S}=\mathrm{RS}$ - R, followed by a division to obtain an image $\mathrm{N}=\mathrm{S} / \mathrm{R}$. The values in $\mathrm{N}$ should be multiplied by a constant to obtain the normalised intensity map. If the signal and reference illumination intensities are equal, the multiplication factor is 1 . The value at the centre of the disc in each image corresponds to the unshifted laser beam frequency, since the velocity here is zero. If the laser frequency remains stable, this value should be constant for each image in the stored series.

The normalised images are quite noisy therefore, when measuring the greyscale level at any position in an image, it was not sufficient to take the value for a single pixel. Instead, an average was taken over a region 10 pixels wide by 10 high centred on the position of interest.

For all pairs of images in each of the two data series, the normalised intensity maps were calculated. Each map was corrected for the variations in signal beam intensity, the correction factor being chosen to produce the expected value for the zero-velocity absorption at the centre of the disc. Figure 6 shows an example of a corrected map from each of the two series, corresponding to the two rotation directions of the disc. An average was then taken of the entire set of 40 normalised images in each series. The resulting images, which are considerably less noisy than the individual images, are shown in figure 7 , again for both rotation directions of the disc. The difficulty in tuning the laser used to a predetermined frequency, has resulted in the unshifted frequency being quite close to the top of the iodine absorption curve during both sets of measurements, and thus the relationship of image intensity to Doppler shift in figure 7 is non-linear. 


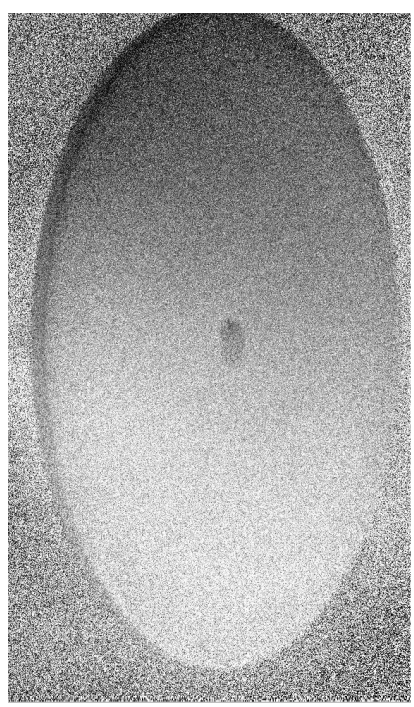

(a)

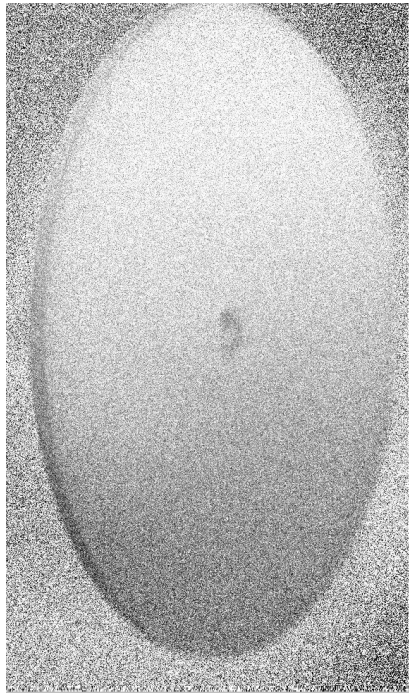

(b)

Figure 6: Normalised intensity maps, corrected for the variation in signal beam intensity for (a) anticlockwise and (b) clockwise rotation of the disc.

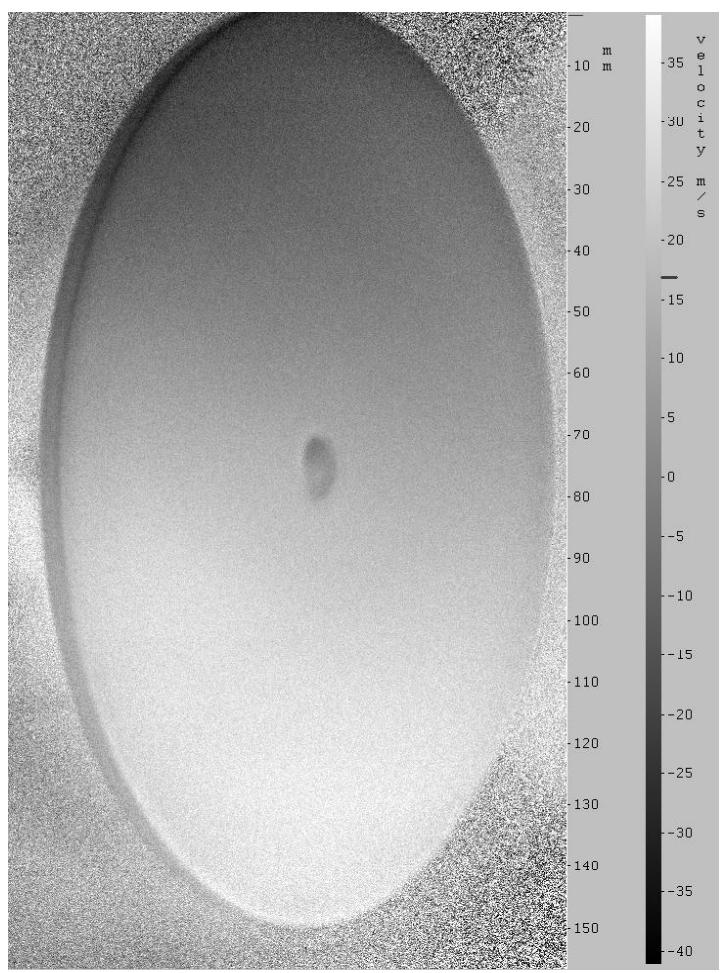

(a)

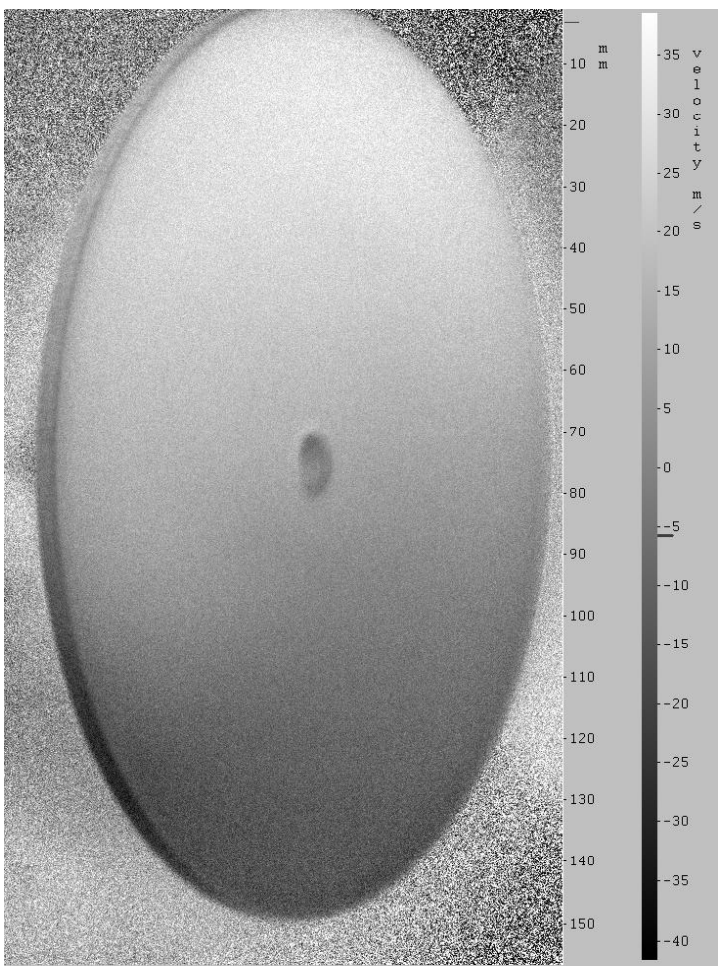

(b)

Figure 7: Normalised intensity maps obtained by averaging over the entire set of 40 images for (a) anticlockwise and (b) clockwise rotation of the disc.

The performance of the PDV system can be assessed by comparison of the data with independent measurements of the disc velocity. Profiles of the greyscale value were taken vertically through the two images of figure 7 close to, but not passing 
through, the centre of the disc. The steel spindle of the motor on which the disc is mounted produces specular reflections. There are small regions where the light from these saturates the camera, causing anomalies in the greyscale level close to the centre of the image. Distortions in the profile therefore arise, but these are avoided by choosing a vertical line at least 15 pixels to one side of the disc centre. Figure 8 (a) shows a profile taken through the averaged image for series A, together with the relevant section of the polynomial fit to the iodine transmission curve, calculated using independent tachometer measurements of disc rotation frequency. The iodine curve is positioned by selecting the transmission on the horizontal centre line of the disc, $75 \mathrm{~mm}$ from the top edge, to match the value determined experimentally.

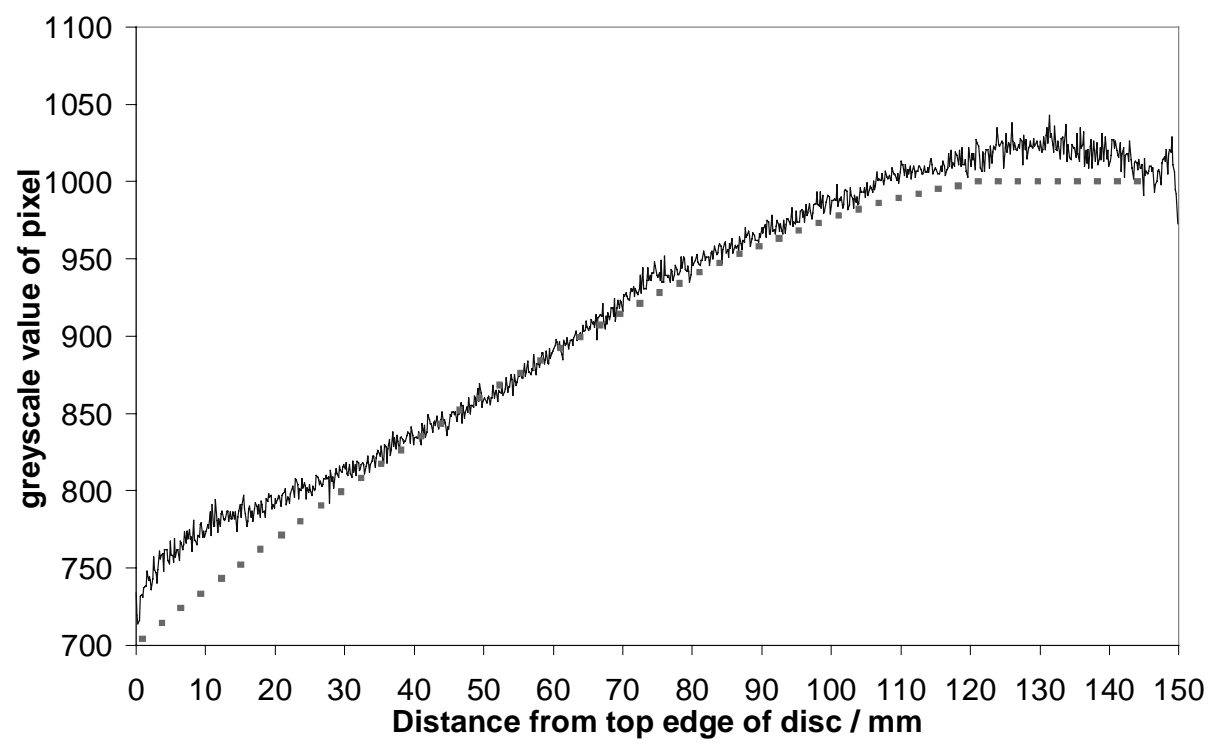

(a)

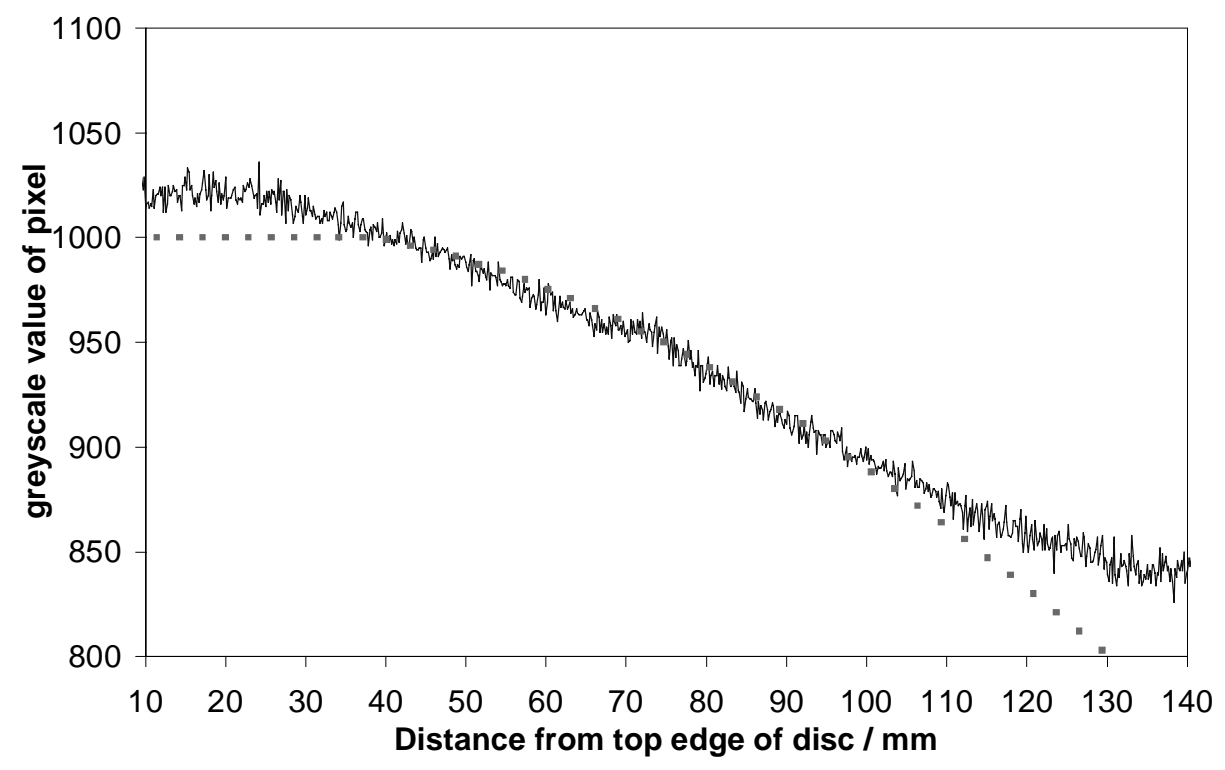

(b)

Figure 8: Intensity profiles taken from the images of figure 7, averaged over 10 columns slightly to the left of the centre of the disc for (a) anticlockwise and (b) clockwise rotation. Solid lines represent the intensity profiles taken from the images, and the dotted lines represent iodine absorption data calculated using independent velocity measurements. 
The PDV measurements match the calculated transmission values very well over the central region of the disc. The maximum error in greyscale value in this region is about $+/-8$, corresponding to a velocity error of $+/-1 \mathrm{~ms}^{-1}$. Towards the edges of the image the fit is less satisfactory, and the experimental values are higher than those expected theoretically, for both the top and bottom edges of the disc. The maximum disparity in greyscale level between the two curves is about 50, corresponding to a velocity error of $7 \mathrm{~ms}^{-1}$. The discrepancy is attributed to low illumination intensity in the outer regions of the disc. The greyscale levels in the original images fall to only about 100 towards the disc edges and, although background levels were subtracted from the images as described above, small variations in background illumination were likely because of the varying signal illumination levels. Any systematic errors will be increased by the signal processing operations and have most effect in regions of low intensity.

The same behaviour is seen in the profile of figure 8 (b), for clockwise rotation of the disc. As expected, the gradient of this curve is negative, rather than positive. Once again, the gradient of the experimental curve is in good agreement with the polynomial fit to the iodine transmission over a central region of the disc. However, the region over which the experimental curve departs from the calculated line is larger and the disparity slightly greater, than in figure 8 (a). This is ascribed to a very slight change in illumination conditions between the two sets of measurements, which was not compensated for by acquisition of a new pair of background images.

\section{DISCUSSION}

The results presented above indicate that the frequency-switching technique can provide PDV velocity data with a resolution of up to $1 \mathrm{~m} / \mathrm{s}$ from a spinning disc. The accuracy and resolution of the information deteriorate in regions of low illumination intensity. This problem is, however, universal in PDV measurements and can be overcome by the use of a more powerful laser and perhaps also the introduction of beam-shaping optics to produce a more uniform illumination beam. Processing is made extremely straightforward in the one camera system, by the avoidance of any pixel-matching requirement. The calculations have been made slightly more complicated in these experiments by the need to correct for variations in signal beam intensity, which were not anticipated. Altering the acquisition procedure to allow the AOM to stabilise before starting acquisition could eliminate this complication.

For the technique to be of real utility it must, of course, be proved in seeded flows. In the next phase of the research programme the intention is to test the system on a ducted airflow, using seeding from a smoke generator. The optical power in the scattered beam is expected to be much lower from a real flow than from the spinning disc. However, the measurement area of interest is often very much smaller than the $150 \mathrm{~mm}$ diameter circle of the disc, so lack of power is not expected to be a major problem. Furthermore, using a different laser, the power in the illumination beam can be increased by an order of magnitude. An additional increase could be achieved by the use of a Pockels cell, synchronised with the AOM, at the laser output, to switch all the available laser power between the two orthogonal linear polarisation states. This would also have the advantage of providing images under either pure reference, or pure signal illumination, thereby avoiding the subtraction stage of the image processing and improving the signal-to-noise performance.

For the camera exposure times used in these experiments, the optical power in the scattered beam remains constant with time, as it is always integrated over at least one complete rotation of the disc. An important difference in a real flow is that the scattered power, and its distribution over the camera image, may change between acquisition of the reference and signal images. In a steady-state flow, if the seeding remains fairly uniform, the errors caused by this should remain small, and can be minimised by taking an average over many normalised images. The number of images required for acceptable performance will depend on the nature of the flow.

In a rapidly time-varying flow, of course, averaging over many images is not an option, and application of the technique is not straightforward. A pulsed laser would probably be required for this type of flow, to freeze the motion of the particles ${ }^{14}$. With state-of-the-art cameras, the time between two frames can be as short as $100 \mathrm{~ns}$, so near-simultaneous acquisition would be possible. This would probably be acceptable for moderate-speed flows. However, the fibre that matches the profiles and directions of the two illumination beams would not transmit the high-power laser pulses without damage. A spatial filter could be used instead, but the flexibility of the system would be greatly reduced. 


\section{CONCLUSIONS}

It has been shown that two closely-spaced optical frequencies, produced by switching an acousto-optic modulator, can form the basis of a common-path, single-camera PDV system. Superposition of the signal and reference images, which is one of the major difficulties in the more conventional two-camera PDV arrangement, is achieved automatically, albeit at the expense of precise simultaneity in the acquisition of the image pair. The performance of the dual frequency system has been assessed using a rotating disc as a test flow and, after a combination of temporal and spatial averaging, a velocity resolution of $+/-1 \mathrm{~ms}^{-1}$ has been achieved.

The potential benefit of the technique is a major simplification of the PDV image-capture system for time-invariant flows. It is most likely to find application for measurements in well-seeded flows, over areas of the order of a few square centimetres, since fibre delivery of the illumination beams limits the maximum power in the measurement volume to a few hundred milliwatts. The use of optical frequencies separated by only $520 \mathrm{MHz}$ effectively eliminates any differences in scattering between the reference and signal beams.

\section{ACKNOWLEDGMENTS}

This work was partially supported by a UK Royal Society grant and a grant from the Engineering and Physical Sciences Research Council (EPSRC), UK.

Thanks are due to Edmon Chehura for calibrating the iodine cell used in the experiments, and providing the plot of cell transmission as a function of laser frequency.

\section{REFERENCES}

[1] Komine, H., Brosnan, S., Litton, A., and Stappaers, E., "Real-Time Doppler Global Velocimetry", AIAA Paper 91 0337, 1991.

[2] Meyers, J.F. and Komine, H., "Doppler Global Velocimetry: A New Way to Look at Velocity", Laser Anemometry, 1, pp. 289-296, 1991.

[3] Meyers, J.F., "Development of Doppler Global Velocimetry as a Flow Diagnostic Tool", Meas. Sci. Technol., 6, pp 769-783, 1995.

[4] Gerstenkorn S. and Luc, P., "Atlas du Spectre d'Absorption de la Molecule d'Iode 14800-2000 $\mathrm{cm}^{-1}$ Complement: Identification des Transitions du Systeme (B-X)", Laboratoire Aime-Cotton, Centre National de la Recherche Scientifique, Orsay, France, 1986.

[5] Chan,V.S.S., Heyes, A.L., Robinson, D.I. and Turner, J.T. "Iodine absorption filters for Doppler global velocimetry", Meas. Sci. Technol. , 6, pp 784-794, 1995.

[6] Ford, H.D. and Tatam, R.P. (1997) "Development of extended field Doppler velocimetry for turbomachinery applications", Optics and Lasers in Engineering, 27, pp 675-696.

[7] Ainsworth,R.W. and Thorpe, S.J., "The development of a Doppler global velocimeter for transonic turbine applications", Int. Gas Turbine and Aeroengine Congress and Exposition (ASME paper 94-GT-146), The Hague, Netherlands, June 13-16 1994

[8] Meyers, J.F., "Doppler Global Velocimetry: The Next Generation?", AIAA Paper 92-3897, Nashville, 1992.

[9] Manners, R.J., Thorpe, S.J. and Ainsworth R.W., "Image processing techniques for Doppler Global velocimetry", Proc. I. Mech. E. Conference on Optical Methods and Data Processing in Heat and Fluid Flow, London, 1996.

[10] Arnette, S.A., Elliott, G.S., Mosedale, A.D, and Carter C.D., "A Two-Color Approach to Planar Doppler Velocimetry", AIAA Paper 98-0507, Reno, 1998.

[11] Rashleigh, S.C., "Origins and Control of polarization effects in single-mode fibers", Journal of Lightwave Technology, LT-1, 312-331, 1983.

[12] Quinn, T.J. and Chartier, J.-M., "A new type of iodine cell for stabilized lasers", IEEE Transactions on Instrumentation and Measurement, 42, 405-406,1993.

[13] Irani, E., and Miller, L. S., "Evaluation of a Basic Doppler Global Velocimetry System", Society of Automotive Engineers, Paper 951427, 1995.

[14] McKenzie, R.L., "Measurement Capabilities of Planar Doppler Velocimetry Using Pulsed Lasers", AIAA Paper 950297, 1995. 